\title{
Quantification of Benzene in Groundwater Using SH-Surface Acoustic Wave Sensors
}

\author{
Florian Bender ${ }^{1}$, Rachel Mohler ${ }^{2}$, Antonio J. Ricco ${ }^{3}$, Fabien Josse $^{1}$ \\ ${ }^{1}$ Department of EECE, Marquette University, Milwaukee, WI 53201-1881, USA, \\ fabien.josse@mu.edu \\ ${ }^{2}$ Chevron Energy Technology Co., 100 Chevron Way, Richmond, CA 94801, USA, \\ ${ }^{3}$ Department of Electrical Engineering, Center for Integrated Systems, Stanford University, Stanford,
} CA 94305, USA

\begin{abstract}
:
A need exists for compact sensor systems capable of in-situ monitoring of groundwater for fuel and oil contamination. The work reported here addresses this need using shear horizontal surface acoustic wave (SH-SAW) sensors, which function effectively in the liquid phase. To achieve enhanced sensitivity and partial selectivity for hydrocarbons, the devices are coated with thin chemically sensitive polymer films. Various polymer materials are investigated with the goal of identifying a set of coatings suitable for a sensor array. The system is tested with compounds indicative of fuel and oil contamination, in particular, BTEX (benzene, toluene, ethylbenzene and xylenes), at relatively low concentrations. Of particular importance is benzene, a known carcinogen. Using responses of the SHSAW sensor devices coated with three different polymer materials, benzene was quantified in the aqueous phase in the presence of other aromatic interferents. It is shown that various concentrations of BTEX in water can be identified and quantified by evaluation of both steady-state and transient response information.
\end{abstract}

Key words: groundwater, fuel and oil contamination, surface acoustic wave sensors, liquid-phase sensing, benzene, transient response information.

\section{Introduction}

Leaks and spills from fuel and oil tanks, pipelines, and other sources may contaminate groundwater, lakes, rivers, and oceans, posing potential threats to public health [1-4]. Timely detection of such contamination can minimize the impact on public health and reduce cleanup costs; periodic monitoring is also sometimes required by law. The current practice (the analysis in the laboratory of field-collected samples [2]), however, is too time-consuming for continuous monitoring. Therefore, compact sensor systems are under development that will allow frequent or continuous, in-situ monitoring of groundwater and bodies of water at critical sites [5].

Sensor systems based on SH-SAWs (shearhorizontal surface acoustic waves) represent a promising approach for this application. SHSAWs can propagate along a surface in contact with a liquid phase without excessive acoustic attenuation [6]. For this work, a $103-\mathrm{MHz} 36^{\circ}$ $\mathrm{YX}-\mathrm{LiTaO}_{3}$ sensor platform was selected [7], [8]. In order to enhance sensitivity and provide partial selectivity for the analytes of interest, the sensors were coated with thin $(\leq 1 \mu \mathrm{m})$ organic polymer films. Many polymers show favorable physical and chemical characteristics for hydrocarbon sensing; they are easy to prepare and offer the possibility of tailoring their chemical structure for a particular application. For these reasons, polymers are by far the most common materials for hydrocarbon sensing [9]. The performance of the sensors and various coating materials has been investigated under varying environmental conditions, including changes in temperature, acidity, and salinity [10].

BTEX compounds (benzene, toluene, ethylbenzene, and xylenes) are present in crude oil and its refined products [11] and are regulated by government agencies. Therefore, these aromatic compounds have been selected as indicators of fuel and oil contamination for the present work. The BTEX compounds can be detected by polymer-coated SH-SAW sensors in liquid phase at low concentrations; for ethylbenzene, a detection limit of about 40 $\mathrm{ppb}(\mu \mathrm{g} / \mathrm{L})$ has been achieved with the current sensor design. 
Among the BTEX compounds, benzene is of particular concern due to its carcinogenicity [12], making it of paramount importance, and in some cases a legal requirement, to quantify benzene contamination in water. This task is challenging, not only because relevant concentrations are in the low ppm (mg/L) to ppb $(\mu \mathrm{g} / \mathrm{L})$ range [5], [12] but also because of frequent co-contamination by similar aromatic compounds including toluene, ethylbenzene, and xylenes. Here we address this challenge by evaluating both the steady-state frequency shift and the time-dependent behavior of the sensor response. In addition, various polymer materials are investigated as sensor coatings based on their partial selectivities for the analytes of interest [13]. A sensor array will eventually be designed for the purpose of benzene detection and quantification in water contaminated with multiple analytes.

As long as the interaction between a polymer and an analyte is non-specific (physisorptive) and analyte concentrations are sufficiently low, the sorption of the analyte into the polymer from aqueous phase can be described by a single partition coefficient [14], and Henry's law can be applied [15] together with the solubility of the analyte. As a result, at low concentrations the response time for sorption of an analyte into a given coating is independent of the analyte concentration in the aqueous phase, and responses to multiple analytes are additive. Because the objective here is to quantify benzene in a mixture, it was critical to first investigate the validity of Henry's law in the concentration range (low ppm to ppb) of interest for the various polymers. It is demonstrated that the response time is specific to each coatinganalyte pair. Binary mixtures of aromatic analytes were next investigated. The resulting response curves were evaluated using dualexponential fits in order to determine benzene concentration in the presence of a single chemical interferent.

\section{Experimental}

The SH-SAW sensor platform used for this work has been previously described in [7] and [8]. The sorbent polymer coatings selected for this study include: poly(ethyl acrylate) (PEA), poly(epichlorohydrin) (PECH), and poly(isobutylene) (PIB) (Sigma-Aldrich). Sorbent polymers were deposited from solution by spin coating and baked for $15 \mathrm{~min}$ at $60^{\circ} \mathrm{C}$, resulting in thicknesses ranging from 0.6 to $1.0 \mu \mathrm{m}$ as indicated below. The sensor platform utilizes a dual delay line design, with the second line coated with poly(methyl methacrylate) (Scientific Polymer Products) and baked for 120 min at $180{ }^{\circ} \mathrm{C}$, resulting in a glassy, non- sorbent coating. The purpose of this reference line is to compensate for the influence of temperature drifts and other secondary effects. All BTEX analytes had purities of $\geq 98.5 \%$ and were purchased from Sigma-Aldrich. The experimental set-up consisted of a network analyzer (Agilent 8753ES) and a switch/control system (Agilent 3499A) to switch between the two SH-SAW delay lines. The sensor was placed inside a liquid flow cell made in-house, and the pump (Eppendorf EVA) was set to a sample flow rate of $0.4 \mathrm{~mL} / \mathrm{min}$. The temperature was held constant at $22.0 \pm 0.1{ }^{\circ} \mathrm{C}$. All data shown are corrected for baseline drift.

\section{Results and Discussion}

Our previous experiments on single analytes at relatively low concentrations ( $\leq 10 \mathrm{ppm}$ ) demonstrated that transient responses can be modeled using a single exponential fit. A characteristic response time constant was found for each coating-analyte combination which, within experimental error, was independent of analyte concentration in the range of interest. As an example, Fig. 1 shows the response of a sensor coated with $0.8 \mu \mathrm{m}$ $\mathrm{PIB}$ to ethylbenzene in the concentration range of $170 \mathrm{ppb}-8 \mathrm{ppm}$.

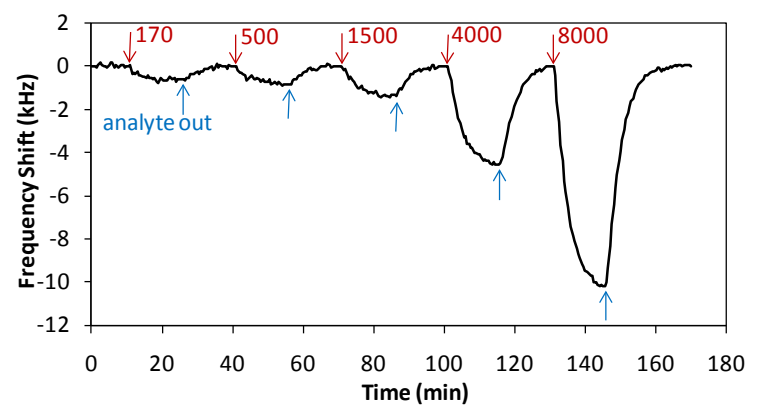

Fig. 1. Response of a SH-SAW sensor coated with $0.8 \mu \mathrm{m}$ PIB to ethylbenzene, added in various concentrations as indicated in the graph (in ppb).

No significant deviation from Henry's law was observed. Therefore, response curves to binary mixtures were modeled using a dualexponential fit of the form:

$$
y(t)=y_{1}\left(1-\exp \left(-t / \tau_{1}\right)\right)+y_{2}\left(1-\exp \left(-t / \tau_{2}\right)\right) .
$$

In Eq. (1), $y(t)$ is the frequency change as a function of time and $\tau_{1,2}$ are the respective response time constants to analytes 1 and 2 obtained from single-analyte measurements. The steady-state frequency shifts, $y_{1,2}$, are proportional to the concentrations of the two analytes in the binary mixture. If the response to each analyte is known from single-analyte measurements, then the analyte concentrations can be readily extracted. Both the values for the measured sensitivity, $S$, (frequency shift/analyte concentration) and $\tau$ are obtained from multiple 
measurements and are listed as average values for various coating-analyte combinations in Tables 1 and 2.

Table 1: Measured sensitivities, $S$ (in $\mathrm{Hz} / \mathrm{ppm}$ ), for three different polymer coatings to various BTEX analytes.

\begin{tabular}{|l|c|c|c|}
\hline Polymer & $S_{\text {benzene }}$ & $S_{\text {toluene }}$ & $S_{\text {ethylb. }}$ \\
\hline $1.0 \mu \mathrm{m}$ PEA & 244 & 690 & 2238 \\
\hline $0.6 \mu \mathrm{m}$ PECH & 109 & 435 & 1450 \\
\hline $0.8 \mu \mathrm{m}$ PIB & 63 & 344 & 1669 \\
\hline
\end{tabular}

Table 2: Measured response times, $\tau$ (in $s)$, for three different polymer coatings to various BTEX analytes.

\begin{tabular}{|l|c|c|c|}
\hline Polymer & $\tau_{\text {benzene }}$ & $\tau_{\text {toluene }}$ & $\tau_{\text {ethylb. }}$ \\
\hline $1.0 \mu \mathrm{m} \mathrm{PEA}$ & 36.1 & 76.7 & 204 \\
\hline $0.6 \mu \mathrm{m}$ PECH & 26.5 & 77.6 & 175 \\
\hline $0.8 \mu \mathrm{m}$ PIB & 29.3 & 84.2 & 245 \\
\hline
\end{tabular}

Fig. 2 shows the frequency responses, $\Delta f$, of a sensor device coated with $1.0 \mu \mathrm{m}$ PEA to two BTEX analytes and their binary mixture. The figure demonstrates that the response to the binary mixture can be modeled using a dualexponential fit with response times, $\tau_{1,2}$, taken from the single analyte responses as indicated by Eq. (1).

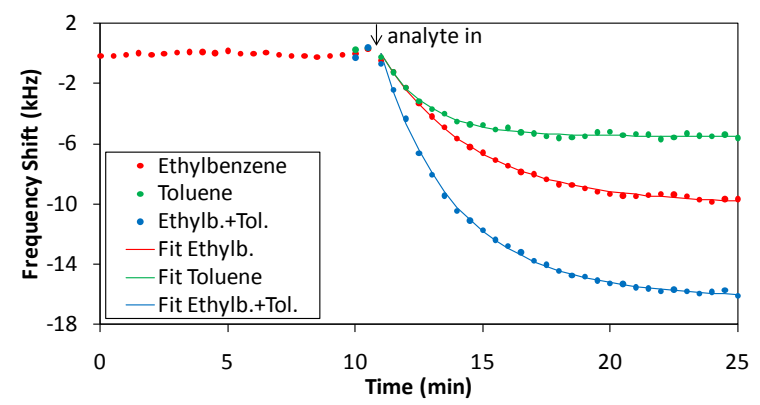

Fig. 2. Response of a SH-SAW sensor coated with 1.0 $\mu \mathrm{m}$ PEA to toluene, ethylbenzene, and their binary mixture, respectively. All concentrations used are $10 \mathrm{ppm}$ (binary mixture: $10 \mathrm{ppm}$ toluene +10 ppm ethylbenzene). Experimental data are modeled with single and dual-exponential fits, respectively.

Fig. 3 shows the frequency response, $\Delta f$, of a sensor device coated with $0.6 \mu \mathrm{m} \mathrm{PECH}$ to several aromatic analytes and binary mixtures. It can be seen that the sensitivities to the analytes fall in the order $S_{\text {ethylbenzene }}>S_{\text {toluene }}>$ $S_{\text {benzene }}$ (for concentrations expressed in ppm). Note that in order to determine the true affinity of a polymer for an analyte, sensitivity has to be normalized to the solubility in the aqueous phase, $C_{S}$, and the molar mass, $M_{W}$, of the analyte:

$\Delta f_{\text {normalized }}=\left(\Delta f / M_{W}\right) /\left(\Delta C_{a m b} / C_{S}\right)$.

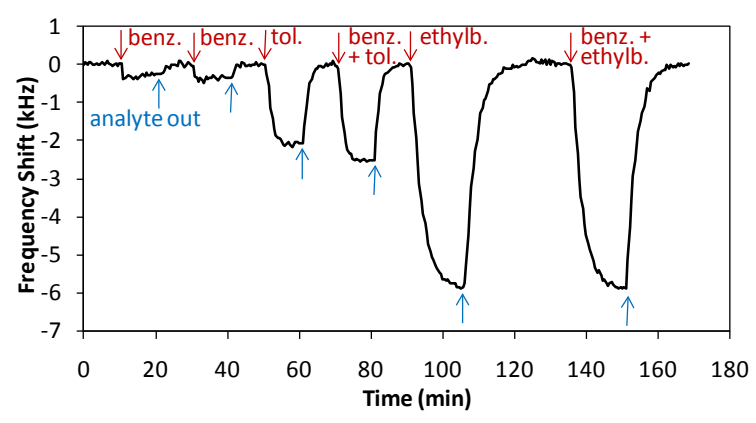

Fig. 3. Response of a SH-SAW sensor coated with $0.6 \mu \mathrm{m} \mathrm{PECH}$ to various single analytes and binary mixtures. Concentrations used are 3 ppm (benzene) and 5 ppm (toluene and ethylbenzene), respectively.

$\Delta C_{a m b}$ is the ambient concentration of the analyte. After normalization, it is seen that the polymers used in this work have a higher affinity for benzene than for the other BTEX compounds [10]. Fig. 3 also shows the different response times for the analytes, benzene being the fastest. For binary mixtures, the transient response is the sum of a faster response (to benzene) and a slower response (second component). The steady-state frequency shifts for single analytes add to give approximately the frequency shift observed for their binary mixture. In order to extract the concentrations of the individual analytes in a binary mixture, response curves to binary mixtures were fit using Eq. (1) and the single-analyte parameters listed in Tables 1 and 2. For the data presented in Fig. 3, the following concentrations were obtained:

- binary mixture of toluene and benzene: 0.9 ppm benzene, 5.8 ppm toluene;

- binary mixture of ethylbenzene and benzene: 1.7 ppm benzene, 4.9 ppm ethylbenzene.

Similar results were obtained for other coating materials and analyte concentrations. The (small) observed errors are expected due to the inaccuracy in analyte concentration in the manual sample mixing procedure. The fact that responses to multiple analytes are additive was independently verified by a different set of experiments. For this purpose, a sensor device was first exposed to $10 \mathrm{ppm}$ of a single analyte. As soon as equilibrium was reached, the device was immediately (i.e., without intermediate flushing with water) exposed to a binary mixture, again containing $10 \mathrm{ppm}$ of the above analyte together with a second analyte. The resulting response to the binary mixture should then show a transient which only reflects the addition of the second analyte since the concentration of the first analyte remains constant. As indicated by the results of Fig. 4, this is indeed the case within experimental error. 


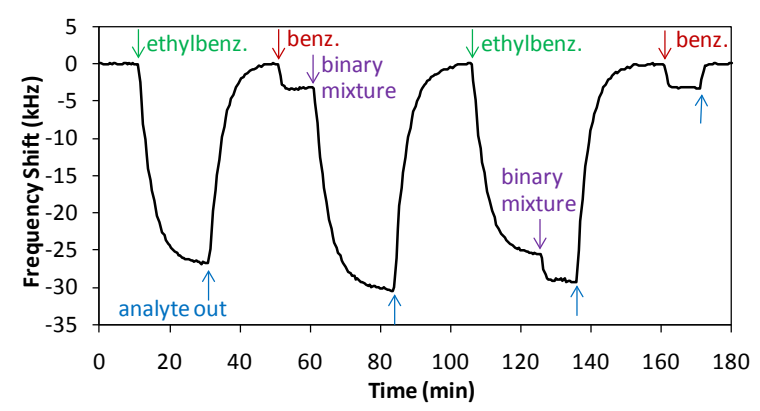

Fig. 4. Response of a SH-SAW sensor coated with 1.0 $\mu \mathrm{m}$ PEA to various single analytes and binary mixtures. All concentrations used are 10 ppm (binary mixtures: 10 ppm benzene +10 ppm ethylbenzene).

\section{Conclusion}

It has been shown that in the concentration range relevant for BTEX detection in groundwater, the responses of polymer-coated SH-SAW sensors to multiple analytes in a mixture are additive, and the response time is specific to each coating-analyte pair. Based on these findings, it has been demonstrated that evaluation of transient responses can help identify and quantify benzene in a mixture. For example, the response to ethylbenzene in Fig. 3 shows about the same frequency shift as the response to the binary mixture of benzene and ethylbenzene, but benzene concentration can still be extracted from the different response time characteristics. Within experimental error, concentrations for ethylbenzene and toluene can usually be predicted with high accuracy while the concentration of benzene, due to its higher solubility in water and lower partition coefficient for the coatings, is quantified with slightly lower accuracy. (It is noted that benzene was easily measurable down to 500 $\mathrm{ppb}$ in water). Therefore, the eventual approach will involve the use of a sensor array utilizing both the transient and the steady-state responses to further enhance the accuracy in benzene quantification.

\section{Acknowledgements}

The authors wish to thank Karen A. Synowiec and Urmas Kelmser from Chevron Energy Technology Co. for helpful discussions.

\section{References}

[1] U.S. Environmental Protection Agency, FY 2010 Annual Report On The Underground Storage Tank Program (2011); EPA-510-R-11-001

[2] Z. Wang, S.A. Stout, M. Fingas, Forensic Fingerprinting of Biomarkers for Oil Spill Characterization and Source Identification, Environ. Forensics 7, 105-146 (2006); doi: $10.1080 / 15275920600667104$

[3] L. Liu, R.X. Hao, S.Y. Cheng, A Possibilistic Analysis Approach for Assessing Environmental
Risks from Drinking Groundwater at PetroleumContaminated Sites, J. Environ. Inf. 2, 31-37 (2003); doi: 10.3808/jei.200300015

[4] M.D. Einarson, D.M. Mackay, Predicting Impacts of Groundwater Contamination, Environ. Sci. Technol. 35, 66A-73A (2001); doi: 10.1021/es0122647

[5] C.K. Ho, A. Robinson, D.R. Miller, M.J. Davis, Overview of Sensors and Needs for Environmental Monitoring, Sensors 5, 4-37 (2005); doi: 10.3390/s5010004

[6] K. Länge, B.E. Rapp, M. Rapp, Surface acoustic wave biosensors: a review, Anal. Bioanal. Chem. 391, 1509-1519 (2008); doi: 10.1007/s00216008-1911-5

[7] F. Josse, F. Bender, R.W. Cernosek, Guided Shear Horizontal Surface Acoustic Wave Sensors for Chemical and Biochemical Detection in Liquids, Anal. Chem. 73, 5937-5944 (2001); doi: 10.1021/ac010859e

[8] Z. Li, Y. Jones, J. Hossenlopp, R. Cernosek, F. Josse, Analysis of Liquid-Phase Chemical Detection Using Guided Shear HorizontalSurface Acoustic Wave Sensors, Anal. Chem. 77, 4595-4603 (2005); doi: 10.1021/ac0504621

[9] B. Pejcic, P. Eadington, A. Ross, Environmental Monitoring of Hydrocarbons: A Chemical Sensor Perspective, Environ. Sci. Technol. 41, 63336342 (2007); doi: 10.1021/es0704535

[10] F. Bender, F. Josse, A.J. Ricco, Influence of Ambient Parameters on the Response of Polymer-Coated SH-Surface Acoustic Wave Sensors to Aromatic Analytes in Liquid-Phase Detection, 2011 Joint Conference of the IEEE IFCS and the EFTF, 422-427 (2011); doi: 10.1109/FCS.2011.5977752

[11] J.S. Arey, P.M. Gschwend, Estimating Partition Coefficients for Fuel-Water Systems: Developing Linear Solvation Energy Relationships Using Linear Solvent Strength Theory To Handle Mixtures, Environ. Sci. Technol. 39, 2702-2710 (2005); doi: 10.1021/es048901l

[12] U.S. Environmental Protection Agency, National Primary Drinking Water Regulations (2009); EPA 816-F-09-004

[13] E.T. Zellers, S.A. Batterman, M. Han, S.J. Patrash, Optimal Coating Selection for the Analysis of Organic Vapor Mixtures with PolymerCoated Surface Acoustic Wave Sensor Arrays, Anal. Chem. 67, 1092-1106 (1995)

[14] Y.K. Jones, Z. Li, M.M. Johnson, F. Josse, J.M. Hossenlopp, ATR-FTIR Spectroscopic Analysis of Sorption of Aqueous Analytes Into Polymer Coatings Used With Guided SH-SAW Sensors, IEEE Sens. J. 5, 1175-1184 (2005)

[15] A. Hierlemann, A.J. Ricco, K. Bodenhöfer, W. Göpel, Effective Use of Molecular Recognition in Gas Sensing: Results from Acoustic Wave and in Situ FT-IR Measurements, Anal. Chem. 73, 30223035 (1999); doi: 10.1021/ac981311 